\title{
Potential biomarkers associated with poor prognosis for locally advanced prostate cancer without lymphatic dissemination
}

\author{
E. Pudova ${ }^{1 *}$, E. Lukyanova ${ }^{1}$, K. Nyushko ${ }^{2}$, S. Kharitonov ${ }^{1}$, A. Snezhkina ${ }^{1}$, \\ M. Fedorova ${ }^{1}$, A. Kaprin ${ }^{2}$, B. Alekseev ${ }^{2}$, A. Kudryavtseva ${ }^{1,2}$ \\ ${ }^{1}$ Engelhardt Institute of Molecular Biology RAS, Moscow, Russia \\ ${ }^{2}$ National Medical Research Radiological Center, Ministry of Health of the Russian Federation, \\ Moscow, Russia \\ *e-mail:pudova_elena@inbox.ru
}

Key words: prostate cancer, prognostic biomarkers, TCGA

Motivation and Aim: Prostate cancer (PC) is a major cause of cancer-related deaths in men worldwide. Locally advanced prostate cancer (LAPC) is characterized by invasion of the prostatic capsule without evidence of nodular or distant metastatic spread. Patients within this clinical category have different risks of recurrence $[1,2]$. Our study is aimed at identifying novel prognostic biomarkers for LAPC, which will lead to optimization of treatment and development of appropriate clinical recommendations.

Methods and Algorithms: We performed bioinformatics analysis of The Cancer Genome Atlas (TCGA) project RNA-Seq data using the computational resources of EIMB RAS "Genome" center (http://www.eimb.ru/rus/ckp/ccu_genome_c.php); 130 samples (only derived from Caucasian patients) of LAPC without lymphatic dissemination were divided into two groups depending on the cancer prognosis (favorable or unfavorable). In the analysis, the genes previously identified by published data as potential prognostic biomarkers were considered.

Results: Additional eight genes, that are characterized by differential expression, were found. Increased expression of TWIST1, TUBB3, and CHAT and decreased expression of CYP1B1, IGSF1, EDN3, MSMB, and SERPINA3 were detected in the group of patients with poor prognosis in comparison to one with favorable prognosis. These genes are involved in the key processes of carcinogenesis, such as angiogenesis, proliferation and migration of tumor cells (CYP1B1, EDN3, TWIST1, TUBB3, and CHAT), as well as with disturbance of regulation of signaling cascades (IGSF1, SERPINA3, and MSMB). Conclusion: Thus, we identified a number of genes as potential prognostic biomarkers associated with poor prognosis of LAPC without lymphatic dissemination.

Acknowledgements: This work was funded by the Program of fundamental research for state academies for 2013-2020 years (No. 1201363819) and by RFBR according to the research project No. 17-29-06083.

\section{References}

1. Nyushko K.M. (2014) Surgical treatment of patients with prostate cancer with a high risk of progression. Oncourology. 2:38-45.

2. Alekseev B.Ya. (2012) Combined treatment of prostate cancer: clinical observations. Oncourology. 4:77-87. 